\title{
UBViz: a software tool for exploring metabolic pathways in 3-D space
}

\author{
Song Li and Hui-Hsien Chou \\ lowa State University, Ames, IA, USA
}

BioTechniques 38:540-542 (April 2005)

For biologists, it is crucial to understand the pathways that describe the relationships and the interactions among molecules functioning in a cell. However, it is difficult for humans to understand a very large graph directly from the raw data, so visualization techniques are required to transform raw data into a more understandable format.

Some approaches of pathway visualization have been proposed previously. Kyoto Encyclopedia of Genes and Genomes (KEGG) (1) provides metabolic pathway data both in XMLformat files and manually created 2-D maps. Although these maps are of very good quality, one obvious drawback is that every change of the pathway may cause considerable effort to redraw the map. KEGG also provides a viewer that automatically draws pathways in 2-D space. However due to the limitation of 2-D layout, in these maps many crossings between lines and texts can be seen, especially when the pathways are complex.

Figure 1 shows a comparison of the visual effectiveness between 2-D and 3-D layout. Figure 1A is a part of the pathway "Pantothenate and $\mathrm{CoA}$ biosynthesis" in 2-D space that is drawn by KEGG's automatic pathway viewer. It is obvious that many lines, nodes, and texts cross each other, which makes it hard for users to understand the relationships among them, whereas in Figure 1B, the same relations in 3-D space is much clearer to understand.

We believe that when the number of nodes and edges in a graph becomes large, it is more preferable to use 3-D technology to visualize it. The advantage of 3-D visualization is that users always have the ability to change the viewpoint to make a better obser- vation. For example, if we draw the same pathway "Pantothenate and CoA biosynthesis" in 3-D space, as shown in Figure 1B, a user can zoom-in and rotate to an appropriate angle in 3-D space so that the links to node C00134 can be seen more clearly.

In the system described by Rojdestvenski (2), a 3-D graph layout algorithm was used on metabolic pathways, and the results were stored in Virtual Reality Modeling Language (VRML) format. Users can download these VRML files and view the 3-D VRML images with a

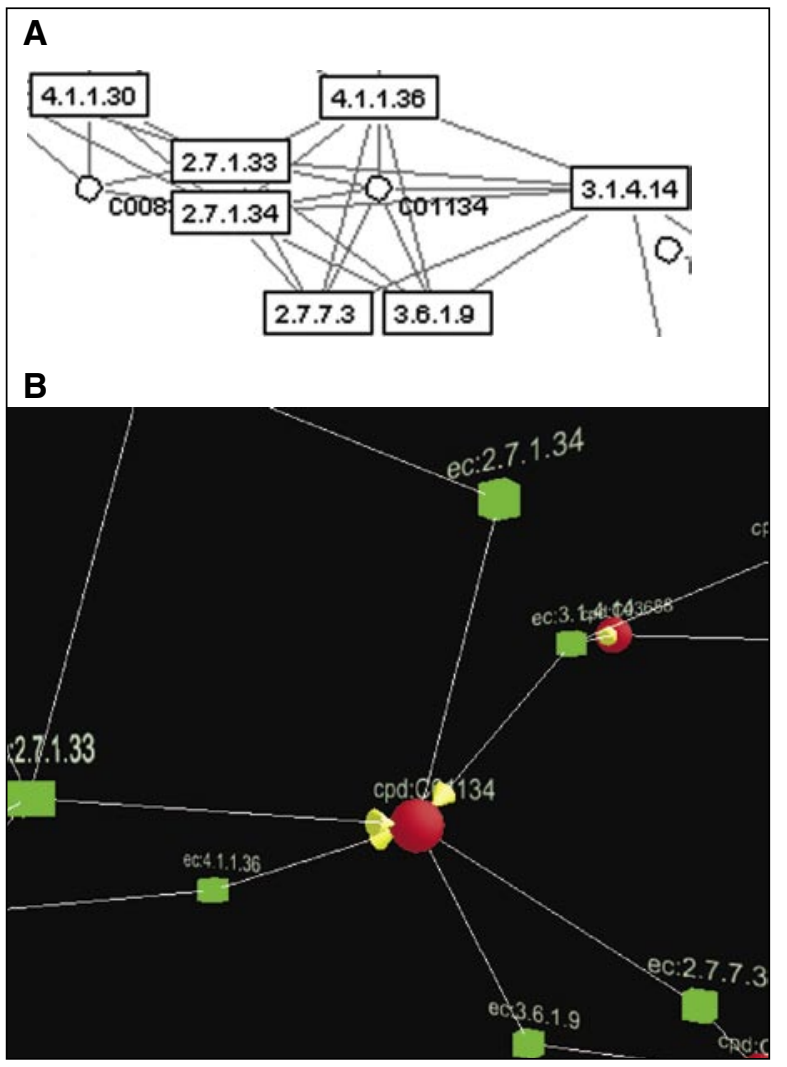

Figure 1. A comparison between 2-D and 3-D visualizations of the same metabolic pathway. (A) Pathway in 2-D space (source: www. kegg.org). (B) The same pathway in 3-D space.
VRML browser. This approach allows data providers to publish 3-D pathway maps on their web site. However, users can only passively receive the data in the visual format that the data providers have already decided.

In this paper, we introduce a novel approach to bringing metabolic pathways into 3-D space. Compared with the aforementioned system, this approach has several advantages. First, our system, UBViz, only needs logical descriptions of the pathways from the data providers and performs the 3-D layout algorithm locally. As a result, less data is needed to be transferred through the network, since the coordinate information is not needed by UBViz. Second, the UBViz's XML interface enables existing XML format pathway files from KEGG to be immediately usable; no change on the KEGG side is needed. Therefore, UBViz's users can make use of all existing database resources, such as the pathway data KEGG provides, and benefit from modern computer 3-D technology immediately without requiring the data providers to expend any effort. Finally, UBViz is a standalone highly efficient $\mathrm{C}++$ program that does not depend on any other software to execute and is easily installable by end-users.

UBViz can use its embedded FTP functionality to download pathway files from KEGG's FTP site directly. Incoming XML files are parsed by a module based on Expat (expat.sourceforge. net). Useful information extracted from the XML files is then kept by UBViz's parser module. This includes $(i)$ a list of nodes and edges that are essential for 3-D layout; (ii) each node's type and name; (iii) information about whether an edge is directed or not, which is used in the map drawing; 


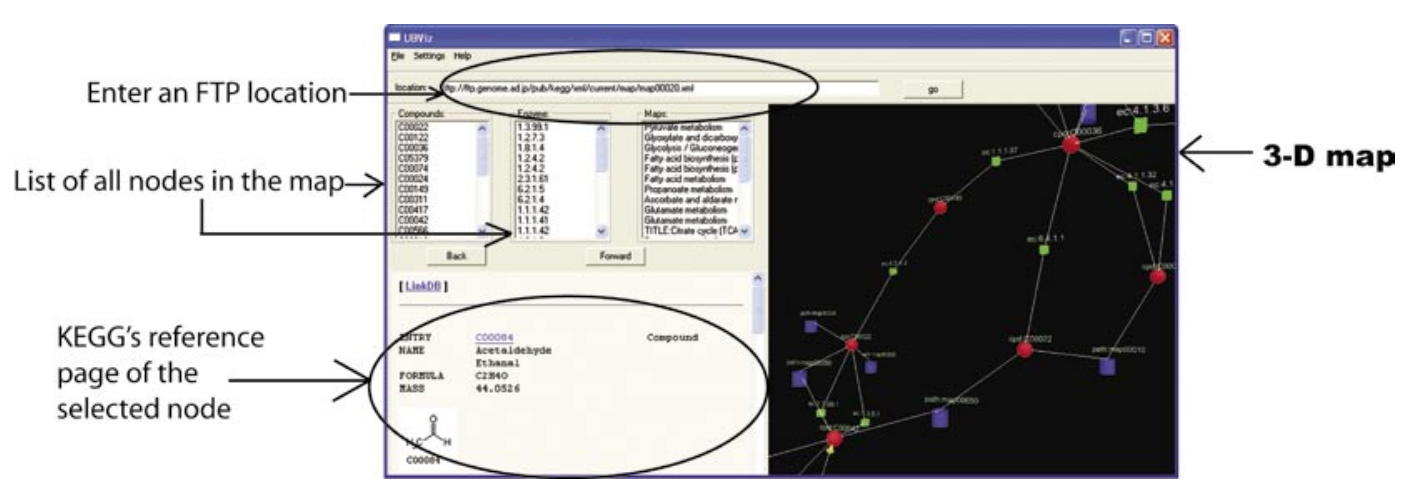

Figure 2. UBViz's user interface.

and (iv) the URL of the reference page for a node, which is used to show the HTML-format explanation of the node. Next, the node and edge information is fed into a 3-D layout module based on GraphViz (3). After the layout computation, 3-D coordinates for each node are generated and are used to draw the objects on the screen. The results are rendered by OpenGL (www.opengl. org). As shown in Figure 2, we use three basic 3-D shapes to represent the three different types of nodes: ( $i$ ) a sphere (red) represents a compound; (ii) a box (green) represents an enzyme; and (iii) a cylinder (purple) represents a link to the connected pathway. For a directed edge, an arrow (yellow) is added to one end of the edge to denote its direction. The viewpoint of the scene can be easily changed by using a set of the 3$\mathrm{D}$ navigation methods implemented in UBViz, which provides adequate power for users to explore the pathways in a virtual 3-D space.

UBViz has a user-friendly graphical interface developed using wxWidgets (www.wxwidgets.org), an open-source multiplatform graphical user interface (GUI) toolkit. As shown in Figure 2, all the users need to know is the name of the metabolic pathway file that UBViz should load. Users can choose to load a file stored in a local disk or to get the file directly from KEGG by inputting the file name in the FTP Location text field. After clicking Go, pathway files will be downloaded and processed in the background. All nodes in the map will be displayed in three list boxes on the left side of the window according to their types, and the 3-D map will be shown on the right side. If users want to know more details about a compound or an enzyme, they can click on the corresponding name in the list boxes. KEGG's reference page will then be shown in the HTML panel below, and the viewpoint of the 3-D map will be automatically adjusted to face the node just selected by the users. Although we have not explored the maximum size of a pathway that can be easily rendered and manipulated in UBViz, the program has successfully handled the largest pathway graph available from the KEGG database, a representation of purine metabolism, which contains 283 nodes and 488 edges.

UBViz is distributed under the Gnu General Public License and can be downloaded from www.complex. iastate.edu/download/UBViz/index. html. UBViz is available on all major computing platforms including Windows, Linux, and $\mathrm{Mac}^{\circledR}$ OS X.

\section{ACKNOWLEDGMENTS}

We thank Jia-Zhen Lee and Yukari Ikuno for the effort they spent on beta-testing UBViz and maintaining UBViz's web pages and Sunyoung Park for graphic design. This project is supported by National Institutes of Health (NIH) grant no. 4R33GM066400.

\section{COMPETING INTERESTS STATEMENT}

The authors declare no competing interests.

\section{REFERENCES}

1.Kanehisa, M. and S. Goto. 2000. KEGG Kyoto encyclopedia of genes and genomes. Nucleic Acids Res. 28:27-30.

2.Rojdestvenski, I. 2003. Metabolic pathways in three dimensions. Bioinformatics 19:24362441

3.Ellson, J., E. Gansner, E. Koutsofios, S. North, and G. Woodhull. 2003. Graphviz and Dynagraph-static and dynamic graph drawing tools, p. 127-148. In M. Junger and P. Mutzel (Eds.), Graph Drawing Software. Springer-Verlag, New York.

Received 1 December 2004; accepted 14 January 2005.

Address correspondence to Hui-Hsien Chou, Departments of Genetics, Development, and Cell Biology and Computer Science, 503 Science II Building, Iowa State University, Ames, IA 50011, USA. e-mail: hhchou@iastate.edu 